\title{
Antibiotic treatment leads to the elimination of Wolbachia endosymbionts and sterility in the diplodiploid collembolan Folsomia candida
}

\author{
Nathan Pike* and Rachel Kingcombe
}

\author{
Address: Department of Zoology, South Parks Road, University of Oxford, Oxford, OX1 3PS, UK \\ Email: Nathan Pike* - nathan.pike.1998@pem.cam.ac.uk; Rachel Kingcombe -r_kingcombe@hotmail.com \\ * Corresponding author
}

Published: 24 August 2009

BMC Biology 2009, 7:54 doi:10.1186/1741-7007-7-54

This article is available from: http://www.biomedcentral.com/174I-7007/7/54

(C) 2009 Pike and Kingcombe; licensee BioMed Central Ltd.

This is an Open Access article distributed under the terms of the Creative Commons Attribution License (http://creativecommons.org/licenses/by/2.0), which permits unrestricted use, distribution, and reproduction in any medium, provided the original work is properly cited.
Received: 26 June 2009

Accepted: 24 August 2009

\begin{abstract}
Background: Wolbachia is an extremely widespread bacterial endosymbiont of arthropods and nematodes that causes a variety of reproductive peculiarities. Parthenogenesis is one such peculiarity but it has been hypothesised that this phenomenon may be functionally restricted to organisms that employ haplodiploid sex determination. Using two antibiotics, tetracycline and rifampicin, we attempted to eliminate Wolbachia from the diplodiploid host Folsomia candida, a species of springtail which is a widely used study organism.
\end{abstract}

Results: Molecular assays confirmed that elimination of Wolbachia was successfully achieved through continuous exposure of populations (over two generations and several weeks) to rifampicin administered as $2.7 \%$ dry weight of their yeast food source. The consequence of this elimination was total sterility of all individuals, despite the continuation of normal egg production.

Conclusion: Microbial endosymbionts play an obligatory role in the reproduction of their diplodiploid host, most likely one in which the parthenogenetic process is facilitated by Wolbachia. A hitherto unknown level of host-parasite interdependence is thus recorded.

\section{Background}

The taxonomic assemblage of endosymbiotic Wolbachia $\alpha$-protobacteria occurs very commonly in insects, mites, crustaceans and nematodes $[1,2]$ and is responsible for a huge variety of reproductive peculiarities. One of the most common of these peculiarities is the incompatibility of crosses between infected males and uninfected females [3], but diverse phenomena including phenotypic male feminisation [4], male killing [5], and thelytokous parthenogenesis [6] have also been conclusively attributed to Wolbachia infections. Folsomia candida (Collembola: Isot- omidae) is a cosmopolitan species of springtail, which is widely used as a standard organism in toxicological assays and as a biological marker of soil pollution $[7,8]$. Although there have been hints that males of the species may exist $[9,10]$, it is generally accepted that the species is made up entirely of females $[8,11]$. Certainly, it is well established that $F$. candida reproduces by thelytokous parthenogenesis for the vast majority of the time. From karyotype studies, we know that $F$. candida uses a diplodiploid (XX/XO) mechanism of sex determination $[11,12]$. F. candida is also host to a strain of Wolbachia which is unique 
from the strains known from other taxa [13]. The effects upon the hosts of this unique supergroup E of Wolbachia are yet to be characterised [14].

It has been argued that the Wolbachia that induces parthenogenesis may be functionally restricted to organisms which have a haplodiploid mechanism of sex determination [15]. The rationale for this argument is that, in haplodiploids, diploidisation of the unfertilised egg is all that is required to achieve sustainable parthenogenesis. Diplodiploid organisms, on the other hand, must also overcome the additional obstacle of inducing eggs to develop (a function usually performed by the fertilising sperm). Whereas we now have many demonstrations of Wolbachia-induced parthenogenesis in haplodiploids (for example, see Stouthamer [15] and Weeks and Breeuwer [16]), to date, we have not been able to obtain good evidence of an equivalent phenomenon in diplodiploid hosts.

We administered the antibiotics tetracycline and rifampicin ad libitum in the yeast food source of $F$. candida (at dry weight doses of $0 \%$ (control), 0.03\%, 0.3\%, 0.9\% and $2.7 \%$ ) over two generations. Elimination of Wolbachia from this host has failed in the past $[13,17]$ but, assuming success, three outcomes were judged possible.

(1) If the Wolbachia are the obligatory agents of parthenogenesis or reproduction, their elimination would render all-female F. candida entirely sterile. The potential for Wolbachia to serve such a critical survival function in the biology of a diplodiploid organism has never been shown.

(2) If $F$. candida possesses an independent mechanism of parthenogenesis and is able to regulate independently reproductive developmental processes, production of females by females could continue even in the absence of Wolbachia (as happens in other organisms, for example, see Matsuura et al. [18]).

(3) If males of $F$. candida do occur (albeit very rarely), elimination of Wolbachia could conceivably result in production of males via a post-zygotic mechanism of sexchromosome elimination known from another (distantly related) collembolan [19]. Of course, the common haplodiploid phenomenon of production of haploid males in the absence of Wolbachia-enforced diploidisation of the nucleus can be ruled out for this diplodiploid species. The discovery of males would raise the possibility of sexual reproduction, with all its genetic consequences, in a species which has hitherto been considered entirely asexual.

\section{Results}

\section{Wolbachia infection}

We randomly selected 10 adult springtails from secondgeneration populations of: (i) the F. candida control treat- ment ( $0 \%$ antibiotic); (ii) the F. candida high-dose (2.7\%) tetracycline treatment; (iii) the F. candida high-dose $(2.7 \%)$ rifampicin treatment; and (iv) the sibling species Folsomia fimetaria, which has males, reproduces sexually, and is reputed not to host Wolbachia. We used Wolbachiaspecific primers in polymerase chain reaction (PCR) analyses of the total DNA extracted from each of these 40 individuals to assess whether the bacteria had been eliminated (see Methods for details of experimental procedures). Wolbachia-specific amplification products were not found in any springtails from the high-dose rifampicin treatment, indicating that this treatment successfully eliminated the bacteria. Similarly, none of the $10 \mathrm{~F}$. fimetaria individuals tested positive for Wolbachia, and it is thus indeed likely that the two are not symbiotic. Perhaps surprisingly, all F. candida individuals from the high-dose tetracycline treatment tested positive for Wolbachia, along with all those from the control populations. The previously reported inefficacy of tetracycline in curing Folsomia of Wolbachia $[13,17]$ is thus supported.

\section{Population parameters}

Clear differences in the population growth rates of highdose rifampicin treatments compared with high-dose tetracycline treatments were also evident, although these differences did not emerge until the second generation (Figure 1). The growth rate of rifampicin populations differed significantly between the two generations $\left(F_{(1,110)}=\right.$ 53.5, $P<0.001)$, because of a second-generation dose effect whereby growth in the $2.7 \%$ and $0.9 \%$ treatments was significantly lower than that in the $0.3 \%$ treatments which, in turn, was lower than those in the $0.03 \%$ or $0 \%$ treatments (Figure 1). No other differences in growth rate among the treatments and/or generations were detectable.

Slight differences in growth rate, clutch size and hatching rate were found among some clones. However, as these differences did not significantly interact with the effects of interest (that is, dose, antibiotic type and generational effects), data have been pooled and clone-specific differences are not presented.

\section{Clutch size}

At week 4 of the first-generation, individuals from the tetracycline treatments had a significantly larger mean clutch size of $44.25 \pm 2.39$ than individuals from the rifampicin treatments, which had a mean clutch size of $33.95 \pm 1.56$ $\left(F_{(1,118)}=10.29, P=0.002\right)$. No dose effect on clutch size was detectable in the tetracycline treatments $\left(F_{4,55}=1.79\right.$, $P=0.15)$. However, clutch size was significantly affected by rifampicin dose $\left(F_{4,55}=7.64, P<0.001\right)$, reaching sizes as low as $27.00 \pm 2.15$ at the highest $2.7 \%$ dose.

Over the 6-month observation period (3 months in which the diet included $2.7 \%$ rifampicin followed by 3 months in which the diet was antibiotic-free), the clutch size of $F$. 


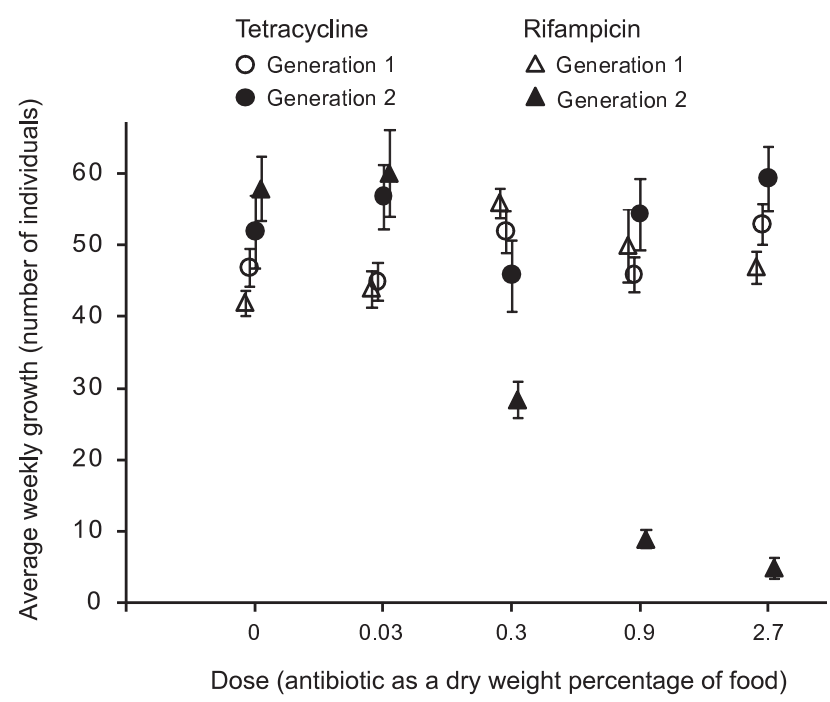

Figure I

Population growth rate (average \pm standard error) measured across four doses of tetracycline and rifampicin over two generations. Note that the $x$-axis is nominal.

candida differed significantly by month $\left(F_{5,24}=5.37, P=\right.$ $0.002)$. While clutch size did not differ among the first 4 months (being $27.45 \pm 1.56$ on average), the sizes observed in the final 2 months $(40.4 \pm 3.37$ and $42.8 \pm$ 2.13 ) were significantly greater than those recorded in the preceding months. In $F$. fimetaria, the average clutch size of $13.03 \pm 0.68$ did not fluctuate significantly across the 6 months.

\section{Hatching rate}

The average hatching rate for clutches laid at week 4 of the first-generation tetracycline treatments was $56.67 \pm$ $3.06 \%$. Hatching rate was not found to be significantly affected by dose in the tetracycline treatments $\left(F_{4,55}=\right.$ $1.82, P=0.14)$. However, dose was significant for hatching rate in the rifampicin populations $\left(F_{4,55}=2.76, P=\right.$ $0.04)$. Hatching rates at week 4 of the first generation fell from around $50 \%$ for doses of $0.03 \%$ and $0.3 \%$ to just $28.2 \pm 3.9 \%$ for the $2.7 \%$ rifampicin dose. Hatching rates were not systematically recorded in the second generation but this rate was observed to decline rapidly to zero in the $2.7 \%$ rifampicin treatments.

\section{Nymph:adult ratio}

Marked demographic changes occurred because of prolonged exposure to high doses of rifampicin. In the first generation, the average nymph:adult ratio (in which higher values indicate a younger, faster-growing population) of the tetracycline treatments was very similar to that of rifampicin treatments $(9.12 \pm 0.31$ versus $8.95 \pm 0.29)$.
Four weeks into the second generation, the average nymph:adult ratio in the tetracycline treatments was $12.31 \pm 0.48$, reflecting the increase expected from young, newly founded populations. In contrast, the nymph:adult ratio in the second generation of rifampicin populations at the same stage had dropped to just $6.96 \pm 0.67$. This treatment difference in week 4 of the second generation was already highly significant $\left(F_{1,118}=42.13, P<0.001\right)$ and it had become absolute by 8 weeks, when nymphs were entirely absent from all the populations exposed to $2.7 \%$ rifampcin and 11 of the 16 populations exposed to $0.9 \%$ rifampicin. The individuals of these populations were both devoid of Wolbachia and totally sterile.

During the 6-month observation period, the nymph:adult ratio remained at 0 for the entire period in F. candida (that is, zero viable nymphs emerged from the hundreds of eggs that were laid). In F. fimetaria, nymphs of all instars were present for all 6 months: the nymph:adult ratio did not differ significantly across months, being $3.53 \pm 0.10$ on average.

\section{Males}

Although males were easily identified in populations of $F$. fimetaria (the sexual species), they were never found in any $F$. candida population.

\section{Discussion}

The elimination of microbial symbionts is likely to be responsible for the sterility observed in $F$. candida. (Although we showed that sustained heavy doses of antibiotic certainly have negative physiological effects, the fact that total sterility persists long after the antibiotic treatment has ended demonstrates that the sterility is unlikely to be due to antibiotic toxicity in itself.) Host sterility is thus attributable to the removal of a microbial strain that facilitates parthenogenesis and/or other reproductive processes. We were careful to distinguish between the facilitation of parthenogenetic diploidisation of the zygote and other non-parthenogenetic reproductive development because it is known that Wolbachia can affect either of these discrete processes. For example, in several Trichogramma wasp species, Wolbachia is responsible for gamete duplication (the parthenogenetic developmental step that leads to a viable zygote) [6], while, in Asobara tab$i d a$, it has the even more fundamental role of facilitating successful oogenesis by inhibiting cell death [20,21]. We can rule out the possibility that Wolbachia plays a role in oogenesis in F. candida because of the continued production of sterile eggs in the face of long-term exposure to rifampicin.

In the current system, we cannot unequivocally attribute the identity of the sterility-associated microbe to Wolbachia: such a definitive identification could only be made 
if we had demonstrated that infection of an uninfected collembolan population with Wolbachia would lead to parthenogenesis and/or restoration of reproductive ability. Nevertheless, there exists excellent evidence to indicate that Wolbachia is the likely culprit. Firstly, we can rule out another common bacterial agent of reproductive alternation, Cardinium, because this symbiont is absent in $F$. candida [22]. In F. candida, Wolbachia bacteria are extremely abundant within both the early oocyte and the embryo, and cytological observations hint that Wolbachia could possibly be involved in producing a diploid zygote through the prevention of the separation of sister chromatids at meiosis II [17]. We thus suggest that the sterility we observed occurs because Wolbachia plays the crucial role of inducing parthenogenesis. An alternative explanation is that $F$. candida is independently able to produce a diploid zygote but that Wolbachia is an obligate mutualist with a crucial role in reproduction, regardless of whether this reproduction is asexual or sexual.

Rifampicin had a negative influence on clutch size in $F$. candida. We interpret this effect to be a consequence of physiological impairments that were not unexpected: rifampicin is known to affect mitochondrial RNA synthesis in eukaryotes at very high concentrations [23]. However, we note that, while clutch sizes increased to normal levels within 1 month of cessation of the rifampicin treatment, total sterility continued. This observation indicates that, while the toxic effects of rifampicin impaired reproductive physiology, these effects are not the cause of sterility. While a similar negative effect on clutch size was not mirrored in F. fimetaria, our casual observations lead us to suggest that the high dose of rifampicin was also physiologically detrimental in this species but that other traits, which were not systematically measured, tended to be affected.

The mode of action of tetracycline is to inhibit protein synthesis by preventing the association of aminoacyltRNA with the bacterial ribosome [24]. The mode of action of rifampicin is to disrupt an even earlier step in the process of protein synthesis: rifampin binds to prokaryotic DNA-dependent RNA polymerase, inhibiting transcription of DNA to messenger RNA and thereby precluding subsequent translation of RNA to proteins [25]. This different mode of action may be the cause of rifampicin's efficacy in curing F. candida of Wolbachia.

Although this study found no evidence for the existence of males of $F$. candida, it remains possible that such males do occur rarely in natural populations [9]. If males are ever found or, indeed, if females of $F$. candida are able to cross with males from a sibling species that is yet to be described [10], F. Frati, personal communication], future experiments may shed valuable light on whether Wol- bachia infection (or its absence) can influence the possibility of sexual reproduction in the extremely common study species that is F. candida.

This newly discovered role for Wolbachia as an obligate component of reproduction in a diplodiploid animal broadens the already diverse repertoire of phenomena that has been created through the co-evolution of these bacteria and their multitude of hosts. The obligate symbiosis between F. candida and Wolbachia is not seen in other diplodiploid hosts such as the termite Reticulitermes speratus, which is able to continue to reproduce by an independent method of parthenogenesis even after it has been cured of Wolbachia [18].

\section{Conclusion}

Examination of the available evidence on the underlying cause of the observed post-antibiotic sterility suggests that, contrary to prior belief, endosymbiont-facilitated parthenogenesis may not be functionally restricted to haplodiploids. Future work will shed light on the exact mechanism by which microbial endosymbionts can enable parthenogenetic reproduction in diplodiploids. The most likely agent of this reproductive facilitation is Wolbachia. The Wolbachia associated with $F$. candida belongs to supergroup $\mathrm{E}$ and the current results give the first indication of what effect this discrete clade of endosymbionts has on its hosts. Future work may even yield evidence that the widespread and adaptable symbionts that are Wolbachia have achieved yet another evolutionary feat: usurpation of sperm's role of inducing an egg to commence embryonic development.

\section{Methods \\ Study species}

Four clonal populations of $F$. candida (each established from a single parthenogenetic female) were used in experiments involving the administration of antibiotics. The founding individuals were collected from different geographically isolated field populations in the UK and France. Populations were fed on a paste of baker's yeast and kept in sealed containers on a substrate of $10 \%$ charcoal, $90 \%$ plaster of Paris at a constant temperature of 21 $\pm 0.2^{\circ} \mathrm{C}$ and a relative humidity of $100 \%$.

The sibling species, Folsomia fimetaria, which reproduces exclusively by sexual means $[17,26]$ was obtained from stock populations maintained at the National Environmental Research Institute, Silkeborg, Denmark. This species was used as a speculative negative control for PCR analyses to detect the presence of Wolbachia. Speculation that this species does not host Wolbachia was based on the observations of Frati et al. [10] that sexually reproducing collembolans that are highly morphologically similar to F. candida are devoid of endosymbiotic Wolbachia. 


\section{Antibiotic treatment protocols}

Two antibiotics, tetracycline (Sigma-Aldrich) and rifampicin (Sigma-Aldrich), which are known to have differing modes of action, were combined with the yeast paste food source and administered ad libitum to experimental populations of $F$. candida. For each antibiotic, five dose treatments (measured in percentage dry weight, antibiotic:yeast) were imposed: $0 \%$ (control), 0.03\%, 0.3\%, $0.9 \%$ and $2.7 \%$. Each of these five dose treatments was replicated across four populations within each of the four clones of F. candida to give a total number of 160 experimental populations. After 4 weeks of antibiotic exposure, 20 first-instar nymphs were taken from each experimental population to give rise to a second generation of 160 experimental populations. These second-generation nymphs were thus guaranteed to be the progeny of mothers that had been exposed to the antibiotic environment for at least 18 days prior to laying.

For all experimental populations from both generations, exact population counts were made on the same day of every week for the first 4 weeks of treatment. Clutch size within each replicate population was estimated by isolating a single adult in a separate rearing container and counting the number of eggs in the first batch of eggs laid. Hatching rate was calculated by observing the proportion of first instar nymphs that emerged from 120 to 150 eggs randomly selected from each replicate population.

Genital examination was used to assess the presence of males. For each population, 20 randomly selected adults were killed and the terminal portion of the abdomen was mounted in lactophenol such that the fifth ventral tergite was clearly visible under $\times 450$ magnification.

A 6-month long observation comparing reproduction in populations of $F$. candida and $F$. fimetaria under exposure to $2.7 \%$ rifampicin was also conducted. For the first 3 months of this 6-month period, five populations (with starting sizes of about 100 individuals) of each species were exposed to rifampicin. (This exposure was a continuation of a rifampicin treatment which had been initiated some 4 months earlier.) For the final 3 months of the 6month period, the antibiotic was no longer included in their food source. Each month, the clutch size of one adult female from each of the eight populations was counted. The proportion of nymphs to adults was also calculated for each population on a monthly basis.

\section{PCR assays for presence of Wolbachia}

For F. candida, 10 randomly selected adults from each of the following three second-generation treatments were subjected to a process of total DNA extraction followed by selective amplification: (i) control (that is, no antibiotic exposure); (ii) exposure to $2.7 \%$ tetracycline; and (iii) exposure to $2.7 \%$ rifampicin. In addition, a fourth group of $10 \mathrm{~F}$. fimetaria adults was also analysed. Each individual was squashed with forceps and incubated at room temperature in $50 \mu \mathrm{l}$ of aqueous buffer $(10 \mathrm{mM}$ Tris, $1 \mathrm{mM}$ ethylenediaminetetraacetic acid and $25 \mathrm{mM}$ sodium chloride), which also contained $0.2 \mathrm{mg} / \mathrm{ml}$ proteinase $\mathrm{K}$. After 30 minutes incubation time, the proteinase $\mathrm{K}$ was inactivated by heating the mixture to $95^{\circ} \mathrm{C}$ for 2 minutes. Two primers named V1 (5'-TTGTAGCCTGCTATGGTATAACT-3') and V6 (5'-GAATAGGTTATGATTTTCATGT$\left.3^{\prime}\right)$, which are specific for the $16 \mathrm{~S}$ rDNA of all known strains of $W$. pipientis [13], were used in the PCR amplifications. Amplification reactions of $25 \mu \mathrm{l}$ final volume were created by adding $2 \mu \mathrm{l}$ from the extraction to a solution containing $250 \mu \mathrm{M}$ of each deoxyribonucleotide triphosphate, $12.5 \mathrm{pM}$ of the two primers and $1 \mathrm{U}$ AmpliTaq DNA polymerase (Applied Biosystems). The amplification profile was that used by Frati et al. [10], and consisted of an initial denaturation at $95^{\circ} \mathrm{C}$ for 2 minutes, followed by 35 cycles of 45 seconds of denaturation at $95^{\circ} \mathrm{C}, 45$ seconds of annealing at $52^{\circ} \mathrm{C}$ and 50 seconds of extensions at $72^{\circ} \mathrm{C}$, with a final extension period of 7 minutes at $72{ }^{\circ} \mathrm{C}$. Electrophoresis of amplification products was conducted on agarose gels containing ethidium bromide.

\section{Abbreviations \\ PCR: polymerase chain reaction;}

\section{Authors' contributions}

NP conceived the research and its design, contributed to the experiments, conducted the molecular assays, undertook data analysis and wrote the manuscript. RK contributed to the research design, undertook the experiments and contributed to the manuscript and data analysis.

\section{Acknowledgements}

We are very grateful to Janeck J. Scott-Fordsmand of the National Environmental Research Institute, Silkeborg, Denmark for providing us with populations of $F$. fimetaria. Francesco Frati of the University of Siena, Italy and John $\mathrm{H}$. Werren of the University of Rochester, USA very kindly gave up their time to advise us on Collembola, Wolbachia and the likely nature of their interactions. David Bass and Helen White-Cooper of the University of Oxford, UK provided expert advice and facilities without which our molecular methods would not have been possible.

\section{References}

I. Stouthamer R, Breeuwer JA, Hurst GD: Wolbachia pipientis: microbial manipulator of arthropod reproduction. Annu Rev Microbiol 1999, 53:7I-I02.

2. Werren JH, Zhang W, Guo RL: Evolution and phylogeny of Wolbachia: reproductive parasites of arthropods. Proc $R$ Soc $B$ Biol Sci 1995, 261:55-7I.

3. Werren JH: Biology of Wolbachia. Annu Rev Entomol 1997 , 42:587-609.

4. Rousset F, Bouchon D, Pintureau B, Juchault P, Solignac M: Wolbachia endosymbionts responsible for various alterations of sexuality in arthropods. Proc $R$ Soc B Biol Sci 1992, 250:91-98.

5. Stevens L, Giordano R, Fialho RF: Male-killing, nematode infections, bacteriophage infection, and virulence of cytoplasmic 
bacteria in the genus Wolbachia. Annu Rev Ecol Syst 200I, 32:519-545.

6. Stouthamer R, Breeuwer JAJ, Luck RF, Werren JH: Molecular identification of microorganisms associated with parthenogenesis. Nature 1993, 361:66-68.

7. International Standards Organization: Soil quality-inhibition of reproduction of Collembola (Folsomia candida) by soil pollutants. Report No ISO I I 267:199(E). Geneva 1999.

8. Fountain MT, Hopkin ST: Folsomia candida (Collembola): a "standard" soil arthropod. Ann Rev Entomol 2005, 50:20I-222.

9. Goto HE: Facultative parthenogenesis in Collembola (Insecta). Nature 1960, 188:958-959.

10. Frati F, Negri I, Fanciulli PP, Pellecchia M, De Paola V, Scali V, Dallai R: High levels of genetic differentiation between Wolbachiainfected and non-infected populations of Folsomia candida (Collembola, Isotomidae). Pedobiologia 2004, 48:46I-468.

II. Hopkin ST: Biology of the Springtails. In Insecta: Collembola Oxford: Oxford University Press; 1997.

12. Kiauta B: Review of the germ cell chromosome cytology of Collembola, with a list of chromosome numbers and data on two species new to cytology. Genen en Phaenen 1970, I3:89-99.

13. Vandekerckhove TTM, Watteyne S, Willems A, Swings JG, Mertens J, Gillis M: Phylogenetic analysis of the 16SrRNA of the cytoplasmic bacterium Wolbachia from the novel host Folsomia candida (Hexapoda, Collembola) and its implications for wolbachial taxonomy. FEMS Microbiol Lett 1999, 180:279-286.

14. Lo N, Casiraghi M, Salati E, Bazzocchi C, Bandi C: How many Wolbachia supergroups exist? Mol Biol Evol 2002, 19:34I-346.

15. Stouthamer R: Wolbachia-induced parthenogenesis. In Influential Passengers: Inherited Microorganisms and Invertebrate Reproduction Edited by: O'Neill SL, Hoffmann AA, Werren JH. Oxford: Oxford University Press; 1997: 102-124.

16. Weeks AR, Breeuwer JAJ: Wolbachia-induced parthenogenesis in a genus of phytophagous mites. Proc $R$ Soc B Biol Sci 200I, 268:2245-225I.

17. Riparbelli MG, Giordano R, Callaini G: Centrosome inheritance in the parthenogenetic egg of the collembolan Folsomia candida. Cell Tissue Res 2006, 326:861-872.

18. Matsuura K, Fujimoto M, Goka K: Sexual and asexual colony foundation and the mechanism of facultative parthenogenesis in the termite Reticulitermes speratus (Isoptera, Rhinotermitidae). Insectes Soc 2004, 5 I:325-332.

19. Dallai R, Fanciulli PP, Frati F: Chromosome elimination and sex determination in springtails (Insecta, Collembola). J Exp Zool 1999, 285:215-225.

20. Dedeine F, Vavre F, Fleury F, Loppin B, Hochberg ME, Boulétreau M: Removing symbiotic Wolbachia bacteria specifically inhibits oogenesis in a parasitic wasp. Proc Natl Acad Sci USA 200I, 98:6247-6252

21. Pannebakker BA, Loppin B, Elemans CPH, Humblot L, Vavre F: Parasitic inhibition of cell death facilitates symbiosis. Proc Natl Acad Sci USA 2007, 104:212-215.

22. Zchori-Fein E, Perlman SJ: Distribution of the bacterial symbiont Cardinium in arthropods. Mol Ecol 2004, 13:2009-2016.

23. Molavi A: Antimicrobials III: sulfonamides, trimethoprim and anti-mycobacterial agents. In Basic Pharmacology in Medicine 3rd edition. Edited by: Di Palma J, Di Gregorio J. New York: McGraw-Hill; 1990.

24. Chopra I, Roberts M: Tetracycline antibiotics: mode of action, applications, molecular biology, and epidemiology of bacterial resistance. Microbiol Mol Biol Rev 200 I, 65:232-260.

25. Campbell EA, Korzheva N, Mustaev A, Murakami K, Nair S, Goldfarb A, Darst SA: Structural mechanism for rifampicin inhibition of bacterial RNA polymerase. Cell 200I, 104:90I-912.

26. Chang SL: Some physiological observations on two aquatic Collembola. Trans Am Microsc Soc 1966, 85:359-37I.

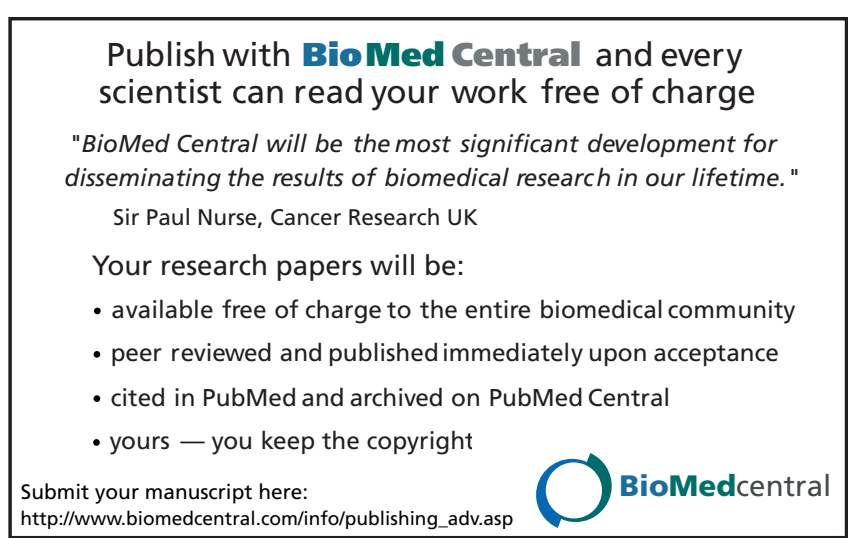

